\title{
Análise exergética de uma planta de digestão anaeróbica alimentada com resíduos da bovinocultura leiteira
}

\author{
Exergy analysis of an anaerobic digestion plant fed with dairy cattle waste \\ Análisis exergético de una planta de digestión anaeróbica alimentada con residuos de ganado lechero
}

Recebido: 28/09/2021 | Revisado: 08/10/2021 | Aceito: 11/10/2021 | Publicado: 12/10/2021

\begin{abstract}
ORCID: https://orcid.org/0000-0001-5285-1286 Universidade Estadual do Oeste do Paraná, Brasil E-mail: victorvaz.fis@gmail.com

Fabiane Mariah Bissani

ORCID: https://orcid.org/0000-0003-3252-9622 Universidade Estadual do Oeste do Paraná, Brasil E-mail: fabianebissani@gmail.com

Eric Valero Carvalho da Silva

ORCID: https://orcid.org/0000-0003-0385-7619 Universidade Estadual do Oeste do Paraná, Brasil E-mail: evcdasilva@gmail.com

Samuel Nelson Melegari de Souza ORCID: https://orcid.org/0000-0002-3581-902X Universidade Estadual do Oeste do Paraná, Brasil E-mail: Samuel.Souza@unioeste.br

Bruna Bissani

ORCID: https://orcid.org/0000-0001-7112-9627 Universidade Estadual do Oeste do Paraná, Brasil E-mail: bissani.b@gmail.com

Juliano de Souza

ORCID: https://orcid.org/0000-0002-6497-1709 Universidade Estadual do Oeste do Paraná, Brasil E-mail: sustentarebiogas@gmail.com
\end{abstract}

Victor Vaz

\section{Resumo}

No intuito de integrar diferentes processos de reaproveitamento de resíduos agropecuários, a produção de biogás e biofertilizante, produzidos pela digestão anaeróbia, são essenciais para o desenvolvimento sustentável e diversificação da matriz energética brasileira. Como a análise do desempenho termodinâmico desses sistemas é escassa na literatura, este trabalho teve por objetivo associar os parâmetros físico-químicos de resíduos da bovinocultura leiteira ao valor de suas respectivas exergia química. E assim, avaliar a eficiência do biodigestor com base em dados operacionais reais da Starmilk, localizada no município de Céu Azul no oeste do Estado do Paraná, Brasil. Os resultados mostram que o conteúdo exergético do dejeto fresco foi de $16,03 \mathrm{MJ} / \mathrm{kg}, 15,35 \mathrm{MJ} / \mathrm{kg}$ para o biofertilizante e 29,55 MJ/m³ $(\mathrm{CNTP}) \mathrm{de}$ exergia do biogás. Com base nas análises feitas, infere-se que o biodigestor analisado possui uma eficiência exergética global de 72,82\%, podendo recuperar 27,29\% da exergia do estrume bovino na forma de biogás.

Palavras-chave: Exergia; Dejetos de gado leiteiro; Produção de biogás; Eficiência termodinâmica; Composição química.

\begin{abstract}
In order to integrate different processes for the reuse of agricultural waste, the production of biogas and biofertilizer, produced by anaerobic digestion, are essential for the sustainable development and diversification of the Brazilian energy matrix. As the analysis of the thermodynamic performance of these systems is scarce in the literature, this work aimed to associate the physicochemical parameters of dairy cattle residues with the value of their respective chemical exergy. And thus, evaluate the efficiency of the biodigester based on real operational data from Starmilk, located in the municipality of Céu Azul in the west of the State of Paraná, Brazil. The results show that the exergy content of fresh manure was $16.03 \mathrm{MJ} / \mathrm{kg}, 15.35 \mathrm{MJ} / \mathrm{kg}$ for the biofertilizer and $29.55 \mathrm{MJ} / \mathrm{m}^{3}$ (CNTP) of biogas exergy. Based on the analyzes carried out, it is inferred that the analyzed biodigester has an overall exergy efficiency of $72.82 \%$, being able to recover $27.29 \%$ of the exergy of cattle manure in the form of biogas.
\end{abstract}

Keywords: Exergy; Dairy cattle manure; Biogas production; Thermodynamic efficiency; Chemical composition. 


\section{Resumen}

Para integrar diferentes procesos de reutilización de residuos agrícolas, la producción de biogás y biofertilizantes, producidos por digestión anaeróbica, son esenciales para el desarrollo sostenible y la diversificación de la matriz energética brasileña. Dado que el análisis del desempeño termodinámico de estos sistemas es escaso en la literatura, este trabajo tuvo como objetivo asociar los parámetros fisicoquímicos de los residuos de ganado lechero con el valor de su respectiva exergía química. Y así, evaluar la eficiencia del biodigestor con base en datos operacionales reales de Starmilk, ubicado en el municipio de Céu Azul en el occidente del Estado de Paraná, Brasil. Los resultados muestran que el contenido de exergía del estiércol fresco fue de 16,03 MJ / kg, 15,35 MJ / kg para el biofertilizante y 29,55 MJ / $\mathrm{m}^{3}$ (CNTP) de exergía de biogás. De los análisis realizados se infiere que el biodigestor analizado tiene una eficiencia exergética global del 72,82\%, pudiendo recuperar el 27,29\% de la exergía del estiércol bovino en forma de biogás.

Palabras clave: Exergía; Residuos de ganado lechero; Producción de biogás; Eficiencia termodinámica; Composición química.

\section{Introdução}

O Brasil é um dos maiores produtores de leito do mundo, com produção estimada em 2019 de 25 bilhões de litros de leite (CONAB, 2020). A maioria do efetivo rebanho leiteiro corresponde a regimes intensivos. Contudo, uma das principais consequências desses sistemas, no quesito ambiental, é a alta concentração de resíduos e a emissão de gases de efeito estufa.

Diante disso, os biodigestores anaeróbicos apresentam-se como tecnologia promissora. Estes são reatores capazes de tratar resíduos orgânicos, oferecendo uma destinação ambientalmente adequada e gerando renda aos produtores rurais, uma vez que o biogás e o digestato, produtos do processo de digestão, podem ser comercializados.

Neste contexto, é interessante deter informações das conversões químicas dos substratos para avaliar precisamente o desempenho do processo. Diversos trabalhos foram propostos, analisando a eficiência da digestão anaeróbia em termos da redução e estabilização de carga orgânica, remoção de sólidos voláteis ou mesmo no rendimento de biogás (Deublein, Steinhauser, 2011), (Kunz et al., 2019), (Vandevivere et al. 2002), (Angonese et al.,2005), (Bueno, 2010), dentre outros.

No entanto, a análise do desempenho termodinâmico desses sistemas é escassa na literatura, no que diz respeito a análise exergética. Desse modo, este artigo avalia o potencial exergético, tendo como foco, a eficiência exergética da digestão anaeróbia de resíduos da bovinocultura leiteira, por meio de um estudo de caso na Fazenda Iguaçu Starmilk.

Nesta perspectiva, este estudo questiona em como quantificar a energia química disponível do processo de digestão anaeróbia, partindo de parâmetros físico-químicos já utilizados na avaliação de águas residuárias e, a partir disso, avaliar a eficiência termodinâmica do processo.

O conceito de exergia, energia disponível ou mesmo disponibilidade é definido como a máxima quantidade de trabalho útil obtida, por meio de um processo reversível, levando um dado sistema a um estado de equilíbrio termodinâmico com o ambiente de referência (Szargut et al., 1988).

A determinação da exergia, partindo de medições direta de propriedades termodinâmicas como Entalpia (H) e Entropia (S), por meio do calorímetro de bomba, é um método confiável, porém, demorado e complicado. Dessa forma, é interessante usar a exergia química padrão, já tabelada, para determinar o potencial exergético do biogás e dos componentes inorgânicos (Kotas, 2013). Além de uma correlação com a Demanda Química de Oxigênio (DQO) para os componentes orgânicos, conforme sugerido por Tai et al. (1986), Khosravi et al. (2013) e Nakashima (2019).

Então, o objetivo deste artigo é associar os parâmetros físico-químicos de resíduos da bovinocultura leiteira ao valor de suas respectivas exergias químicas. Para tanto, adaptou-se a metodologia proposta por Souza et al. (2018), o qual avalia o potencial exergético da vinhaça a partir de parâmetros físico-químicos já utilizados na avaliação de resíduos sólidos. 


\section{Materiais e Métodos}

\subsection{Coleta da Amostra}

O experimento, com coleta de dados primário foi realizado na Fazenda Iguaçu Starmilk, situada no município de Céu Azul - PR. Na latitude $25^{\circ} 02^{\prime} 08$ Sul longitude 534' 48 Oeste, com altitude média de 620 metros. O clima da região é Subtropical Úmido Mesotérmico com temperaturas médias anuais de $19,1^{\circ} \mathrm{C}$ (Nitsche et al., 2019).

As amostras coletadas em campo foram encaminhadas para análise no Laboratório do Núcleo de Biotecnologia da Universidade Estadual do Oeste do Paraná (UNIOESTE), Campus de Toledo (PR). Em cada amostragem, foram registradas todas as informaç̃es, como número de identificação da amostra, data e hora, condições meteorológicas nas últimas 24 horas e temperatura no instante da coleta. E para conservar a integridade do material, as amostras foram mantidas a $4{ }^{\circ} \mathrm{C}$.

Além disso, as amostras de entrada (afluente) e saída (efluente) foram coletadas numa área com chegada frequente de resíduo líquido, evitando um lodo saturado. Também foram coletados, nas tubulações do gasômetro, $40 \mathrm{ml}$ de biogás, com o auxílio de seringas de $20 \mathrm{ml}$.

Para estimativa da exergia química do biogás, foram medidas as concentrações de metano $\left(\mathrm{CH}_{4}\right)$, dióxido de carbono $\left(\mathrm{CO}_{2}\right)$, monóxido de carbono $(\mathrm{CO})$ e Sulfeto de hidrogênio $\left(\mathrm{H}_{2} \mathrm{~S}\right)$ pelo equipamento portátil de extração de gases Landtec gem 5000 .

A composição do biogás também foi determinada por cromatografia gasosa em cromatógrafo Shimadzu® 2014

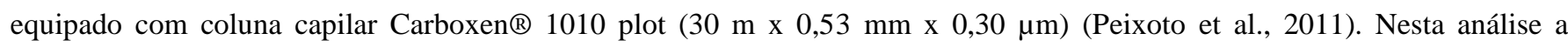
composição da amostra é determinada pela normalização da área de pico de cada gás com a área do pico do gás puro (padrão).

Para este estudo foram considerados, como parâmetro complementares, os dados disponibizados pelo CIBiogás referente aos anos de 2016 a 2018. Segundo esses dados, o efluente produzido na Starmilk, gera cerca de $200 \mathrm{~m}^{3}$ resíduos por dia. Estes resíduos são conduzidos para um biodigestor de fluxo ascendente, de $3500 \mathrm{~m}^{3}$ e cumprindo um Tempo de Retenção Hidráulica (TRH) de 17,5 dias, produz em média 600 m³/dia de biogás, nas condições normais de temperatura e pressão (CNTP), gerando até $840 \mathrm{kWh} /$ dia com uma potência instalada do gerador de $230 \mathrm{~kW}$ (Biasi et al.,2018).

\subsection{Caracterização do resíduo bovino}

A biomassa de entrada é constituída por uma mistura de água, fezes, urina, resto de alimento e maravalha (utilizada como cama animal). Como este resíduo possui uma alta variabilidade, algumas generalizações serão consideradas para representar sua composição.

Nesse sentido, a Demanda Química de Oxigênio (DQO) está associada a concentrações de matéria orgânica presente na biomassa. E o Nitrogênio amoniacal $\left(\mathrm{N}_{-} \mathrm{NH}_{4}\right)$, Pentóxido de Fósforo $\left(\mathrm{P}_{2} \mathrm{O}_{5}\right)$, Óxido de Potássio $\left(\mathrm{K}_{2} \mathrm{O}\right)$, Óxido de Cálcio $(\mathrm{CaO})$ e Óxido de Sódio $\left(\mathrm{Na}_{2} \mathrm{O}\right)$ são os representativos dos compostos inorgânicos, declarados como óxidos conforme sugerido por Font-Palma (2019).

A medição desses parâmetros físico-químicos, seguem os métodos oficiais de análise da "Association of Official Agricultural Chemist" (AOAC), 20ª ed. (2016). A Tabela 1 ilustra os parâmetros utilizados, com seus respectivos métodos especificados. 
Tabela 1 - Parâmetros analisados no ensaio da amostra.

\begin{tabular}{ccccc}
\hline & & \multicolumn{2}{c}{ Valor Experimental } \\
Parâmetros & Unidades & Afluente & Efluente & Método utilizado \\
& & & & \\
\hline DQO & $\mathrm{mg} / \mathrm{l}$ & $22.141,50$ & $10.660,41$ & Colorimétrico \\
Nitrogênio total & $\mathrm{mg} / \mathrm{l}$ & $3.684,67$ & $4.073,60$ & Kjeldahl \\
$P_{2} \mathrm{O}_{5}$ & $\mathrm{mg} / \mathrm{l}$ & $2.826,48$ & $2.320,03$ & Colorimétrico \\
$\mathrm{K}_{2} \mathrm{O}$ & $\mathrm{mg} / \mathrm{l}$ & $18.596,49$ & $9.453,13$ & Fotômetro de chama \\
$\mathrm{CaO}$ & $\mathrm{mg} / \mathrm{l}$ & $3.751,77$ & $2.720,05$ & Oxidimétrico \\
$N a_{2} \mathrm{O}$ & $\mathrm{mg} / \mathrm{l}$ & $1.196,92$ & 708,52 & Titulação de Mohr \\
$N-N H_{4}$ & $\mathrm{mg} / \mathrm{l}$ & $2.474,82$ & $3.425,06$ & Titulométrico \\
Densidade & $\mathrm{kg} / \mathrm{m}^{3}$ & 943,20 & 937,20 & Massa/volume \\
Sólidos Totais & $\%$ & 2,06 & 1,03 & Gravimétrico \\
Sólidos Fixos & $\%$ & 2,23 & 3,49 & Gravimétrico \\
Totais & $\%$ & 7,77 & 6,51 & Gravimétrico \\
Sólidos Voláteis & $\%$ & 7,30 & 7,00 & Eletrométrico \\
Totais & - & &
\end{tabular}

Fonte: Elaboração própria.

\subsection{Análise exergética}

\subsubsection{Cálculo da exergia total}

O cálculo da exergia total de um dado processo é baseado nos componentes: físicos $\left(b_{p h}\right)$, químicos $\left(b_{c h}\right)$, potenciais $\left(b_{P}\right)$ e cinéticos $\left(b_{K}\right)$, conforme exposto na equação (1):

$$
b_{\text {total }}=b_{p h}+b_{c h}+b_{P}+b_{K}
$$

No entanto, a análise de exergia deste estudo considera apenas o componente químico, visto que outras interações de trabalho, como a física, potencial e cinética são desprezíveis em comparação.

A contribuição da exergia física, em especial, foi considerada desprezível pelo fato de os biodigestores analisados não serem aquecidos, não tendo, portanto, variações expressivas de temperatura com relação ao ambiente de referência.

\subsubsection{Exergia química da matéria inorgânica e biogás}

A exergia química do dejeto bovino e biogás foram calculadas partindo da equação (2), conforme demonstrado por Kotas (2013) e proposto por Szargut et al. (1988). Vale destacar que na análise da exergia química dos componentes inorgânicos do dejeto bovino foram considerados em termos do teor de cinzas, no qual é representado pela concentração de sólidos Fixos (SF).

$$
b_{\text {chinorg. }}^{\circ}\left[\frac{k J}{m o l}\right]=\operatorname{SF}[\%] * \sum_{i=1}^{n}\left[x_{i} b_{c h}^{\circ}+R T_{0} x_{i} \ln \left(\gamma_{i} x_{i}\right)\right]
$$

Onde SF são os sólidos fixos [\%], $x_{i}$ é a fração molar, $b_{c h}^{\circ}$ é a exergia química padrão $[\mathrm{kJ} / \mathrm{mol}]$ e $\gamma_{i}$ é o coeficiente de atividade que indica o quanto uma solução não-ideal desviam da idealidade.

Tanto os dejetos bovinos, quanto o biogás foram considerados como uma solução ideal, nesse caso o coeficiente de atividade $\left(\gamma_{i}\right)$ é igual a $1 \mathrm{e}$ as exergias químicas ficam determinadas pelas equações (2.1) e (2.2). 


$$
\begin{aligned}
& b_{\text {ch }}^{\circ} \text { Substrato\&Digestato }\left[\frac{\mathrm{kJ}}{\mathrm{mol}}\right]=\mathrm{SF}[\%] * \sum_{i=1}^{n}\left[x_{i} b^{\circ}{ }_{c h_{i}}+R T_{0} x_{i} \ln \left(x_{i}\right)\right] \\
& i=1 \text {, Nitrogênio amoniacal }\left(\mathrm{N}-\mathrm{NH}_{4}\right) \text {; } \\
& i=2 \text {, Pentóxido de fósforo }\left(P_{2} O_{5}\right) \text {; } \\
& i=3 \text {, Óxido de Potássio }\left(K_{2} O\right) \text {; } \\
& i=4 \text {, Óxido de Cálcio ( } \mathrm{CaO} \text { ); } \\
& i=5 \text {, Óxido de Sódio }\left(\mathrm{Na}_{2} \mathrm{O}\right) \text {. } \\
& b_{{ }_{\text {ch }} \text { biogás }}\left[\frac{k J}{m o l}\right]=\sum_{j=1}^{n}\left[x_{j} b_{c h_{j}}^{\circ}+R T_{0} x_{j} \ln \left(x_{j}\right)\right] \\
& j=1, \operatorname{Metano}\left(\mathrm{CH}_{4}\right) \text {; } \\
& j=2 \text {, Dioxido de Carbono }\left(\mathrm{CO}_{2}\right) \text {; } \\
& j=3 \text {, Monóxido de Carbono ( } \mathrm{CO} \text { ); } \\
& j=4 \text {, Sulfeto de Hidrogênio }\left(H_{2} S\right) \text {; } \\
& j=5 \text {, Amônia }\left(\mathrm{NH}_{3}\right) \text {. }
\end{aligned}
$$

Os cálculos dos componentes inorgânicos e biogás, equação (2.1) e (2.2), foram feitos assumindo a exergia química padrão $\left(b^{\circ}{ }_{c h}\right)$ já tabeladas, proposta por Szargut et al. (2007), com temperatura e pressão de referência de 298,15 K e 1 atm.

As frações molares dos componentes $\left(x_{i}\right)$ do estrume e biogás $\left(x_{j}\right)$ foram calculadas a partir do quociente da molaridade do componente $\left(M_{i}\right)$ pela molaridade total $\left(M_{\text {total }}\right)$. A molaridade dos componentes depende, por sua vez, da concentração comum $\left(C C_{i}\right)$, medida experimentalmente, dividida pela massa molar $\left(M M_{i}\right)$, conforme expresso nas equações (3) e (4).

$$
\begin{aligned}
M_{i} & =\frac{C C_{i}}{M M_{i}} * 10^{3} \\
x_{i} & =\frac{M_{i}}{M_{\text {total }}}
\end{aligned}
$$

Como as concentrações dos componentes do biogás são expressos em porcentagem, sendo necessário converter em g/l por meio da densidade $\left(\rho_{B g}\right)$. A densidade, por sua vez, é calculada por base na massa molar de cada componente, devidamente normalizada, pelo volume molar dos gases perfeitos, conforme equação (5) e (5.1). Ademais, como o monóxido de carbono (CO) e o Sulfeto de hidrogênio $\left(\mathrm{H}_{2} \mathrm{~S}\right)$ foram medidos em parte por milhão $(\mathrm{ppm})$, então, foi realizando a devida conversão em porcentagem, conforme sugerido por Lorimor et al. (2004).

$$
\begin{gathered}
M M_{B g}=\%_{C H_{4}} * M M_{C_{4}}+\%_{C O_{2}} * M M_{C O_{2}}+\%_{C O} * M M_{C O}+\cdots \\
+\%_{O_{2}} * M M_{O_{2}}+\%_{H_{2} S} * M M_{H_{2} S}+\%_{N_{3}} * M M_{N H_{3}} \\
\rho_{B g}\left[\frac{g}{l}\right]=\frac{\left(M M_{B g}\right)}{22,4}
\end{gathered}
$$

\subsubsection{Exergia química da matéria orgânica}

O valor aproximado de exergia dos componentes orgânicos $\left(b_{c h}\right.$ org. $)$ foi avaliada pela correlação com a Demanda Química de Oxigênio (DQO), proposta por Nakashima \& Oliveira (2019) e sendo expressa por (6).

$$
b_{\text {ch }} \text { org. }\left[\frac{J}{l}\right]=14,46 D Q O\left[\frac{m g O_{2}}{l}\right]
$$

Como estamos interessados no valor da exergia específica, dada em $\mathrm{kJ} / \mathrm{kg}$, é necessário dividir o resultado da eq. (6) pela concentração de sólidos totais (ST) presentes nos dejetos. Assim, a equação (6), se torna: 


$$
b_{\text {ch }}\left[\frac{k J}{k g}\right]=\frac{b_{\text {ch }} \text { org. }\left[\frac{J}{l}\right]}{S T\left[\frac{g}{l}\right]}
$$

O método elaborado por Peduzzi et al. (2016) e Shieh \& Fan (1982), foram utilizados para validar os resultados deste trabalho. Estes métodos consistem em representar as propriedades termodinâmicas da biomassa, dentre elas a exergia química, em função da composição elementar (C, H, O, N, S). Já as análises elementares médias dos dejetos de bovinos, foram coletados nos trabalhos de Itoh et al. (2019), Chen et al. (1985), Choi et al. (2014) e Wang et al. (2011), conforme apresentado na tabela (5).

\subsubsection{Eficiência exergética}

É importante destacar que para descrever o desempenho do biodigestor, os cálculos foram baseados em um dia de operação. Além disso, o fluxo de massa do afluente ( $\dot{m}_{\text {afluente }}$ ) e efluente tratado ( $\dot{m}_{\text {efluente. }}$ ), foram calculados multiplicando os $200 \mathrm{~m}^{3}$ de resíduos pelo teor de sólidos totais (ST), conforme equações (7) e (8).

$$
\begin{aligned}
& \dot{m}_{\text {afluente }}\left[\mathrm{m}^{3}\right]=200 * S T_{\text {afluente }}[\%]=4,12 \\
& \dot{m}_{\text {efluente }}\left[\mathrm{m}^{3}\right]=200 * S T_{\text {efluente }}[\%]=2,06
\end{aligned}
$$

Esta consideração é importante, pois a exergia química associada à água não é considerada produto do sistema. Portanto, todos os valores estão em base seca.

Utilizando as equações (2.1) e (2.2) e (6.1), foi proposto o balanço exegético do biodigestor para, posteriormente, avaliar a eficiência exegética do processo, bem como, a eficiência de produção de biogás e estabilização do digestato.

Esse conceito de eficiência exergética, representa o limite teórico de qualidade termodinâmica dos processos (Lozano, Valero 1993). Sua definição básica e inicial, demanda a comparação entre o fluxo de exergia de saída $\left(\dot{B}_{\text {saída }}\right)$ pelo fluxo de exergia de entrada ( $\dot{B}_{\text {entrada }}$ ), podendo ser expressa pela equação (9), mantendo ambas com unidades equivalentes:

$$
\eta=\frac{\dot{B}_{\text {saída }}}{\dot{B}_{\text {entrada }}}=1-\frac{\mathrm{I}}{\dot{B}_{\text {entrada }}}
$$

Onde I é a irreversibilidade do sistema.

Dessa forma, a eficiência global do processo será dada por:

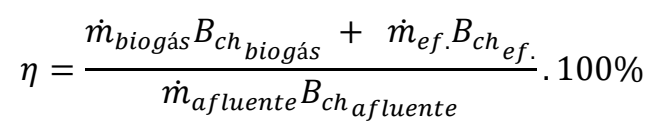

No entanto, esta equação (9.1) não fornece uma caracterização adequada da eficiência termodinâmica de produção de biogás. Isso porque a exergia do efluente, para este nível de agregação, não é utilizável, ou seja, uma exergia desperdiçada no meio ambiente. Desse modo, a eficiência exergética de produção de biogás, considera apenas que:

$$
\eta=\frac{\dot{m}_{\text {biogás }} B_{\text {ch }} \text { biogás }}{\dot{m}_{\text {afluente }} B_{\text {ch }} \text { afluente }} .100 \%
$$

O mesmo pode ser dito quanto a eficiência de estabilização do digestato, que no caso leva em conta apenas a exergia do efluente tratado pela exergia do afluente, conforme equação abaixo (9.3):

$$
\eta=\frac{\dot{m}_{\text {efluente. }} B_{\text {ch }} \text { efluente }}{\dot{m}_{\text {afluente }} B_{\text {ch }} \text { afluente }} * 100 \%
$$

Todas as análises foram desenvolvidas utilizando o software Microsoft Excel, o que possibilitou uma modelagem matemática simples, rápida e acessível. 


\section{Resultados e Discussão}

\subsection{Caracterização físico-química}

Os resultados médios analíticos obtidos na caracterização do dejeto fresco e tratado da bovinocultura leiteria, estão ilustrados na Tabela 1. Estes mostram que os componentes inorgânicos (N, P, K, Ca e Na) estão dentro do intervalo de valores presentes na literatura, conforme relatados em Manitoba (2015) Font-Palma (2019) e Campos et al. (2003). Enquanto que os valores de DQO foram próximos aos dados divulgados pelo CIBiogás nos anos de 2016 a 2017 (valor médio afluente 22.251,67 e efluente 10.142,80 $\mathrm{mg} \mathrm{O}_{2} / \mathrm{L}$ ) coletados na Fazenda Starmilk.

Podemos observar que o conteúdo de sólidos voláteis (SV) foi menor para as amostras digeridas do que para o dejeto fresco, com redução percentual de $16,74 \%$. Isto se deve ao fato de que o processo de digestão anaeróbia transforma biologicamente a matéria orgânica do esterco em biogás, reduzindo o material volátil (Tambone et al., 2009). Essa tendência também foi corroborada pelo aumento na quantidade de sólidos fixos no digestato, uma vez que a matéria orgânica se mineraliza durante a biodigestão (Kobayashi et al., 2013). Isto resulta em um maior conteúdo de componentes inorgânicos presentes nas cinzas do efluente tratado, o que diminui sua energia disponível, dificultando sua utilização em processos de combustão ou pirólise (Lopez et al., 2015).

Verifica-se que o pH do estrume bovino é aproximadamente neutro, indo de 7,3 para 7,0. Isso implica que a maior parte do nitrogênio amoniacal medido está na forma iônica $\left(\mathrm{NH}^{+}\right)$(Kunz et al, 2019). Por esse motivo, na análise exegética dos compostos inorgânicos, utiliza-se a taxa de conversão de 1,29, transformando $\mathrm{N}-\mathrm{NH}_{4}$ em $\mathrm{NH}_{4}^{+}$, conforme sugerido por Lorimor et al. (2004).

\subsection{Conteúdo Exergético das amostras}

A Tabela 2, demonstra as frações molares dos parâmetros físico-químicos, calculados a partir das equações (3) e (4). Com base nesses dados, notou-se um o aumento na fração molar do amônio $\left(\mathrm{NH}_{4}^{+}\right)$, uma pequena diminuição dos teores de óxido de potássio e praticamente nenhuma redução do pentóxido de fósforo $\left(\mathrm{P}_{2} \mathrm{O}_{5}\right)$, óxido de cálcio $(\mathrm{CaO})$ e óxido de sódio $\left(\mathrm{Na}_{2} \mathrm{O}\right)$. $\mathrm{O}$ aumento na concentração de nitrogênio amoniacal está dentro do esperado, pois o nitrogênio na forma orgânica é mineralizado durante o processo de biodigestão. Já os demais componentes não sofrem alteração, mas sim uma precipitação físico-química, conforme relatado por Manitoba (2015).

Tabela 2 - Frações molares em base seca dos componentes inorgânicos do afluente e efluente tratado.

\begin{tabular}{cccccc}
\hline \multirow{2}{*}{ Parâmetros } & $\begin{array}{c}\text { Massa Molar } \\
{[\mathbf{g} / \mathbf{m o l}]}\end{array}$ & \multicolumn{2}{c}{ Molaridade $\left(\boldsymbol{M}_{\boldsymbol{i}}\right)[\mathbf{m o l} / \mathbf{L}]$} & \multicolumn{2}{c}{ Fração Molar $\left(\boldsymbol{x}_{\boldsymbol{i}}\right)$} \\
& Afluente & Efluente & Afluente & Efluente \\
\hline $\mathrm{NH}_{4}^{+}$ & 18,04 & 137,20 & 189,88 & 0,31 & 0,52 \\
$\mathrm{P}_{2} \mathrm{O}_{5}$ & 141,94 & 19,91 & 16,35 & 0,05 & 0,04 \\
$\mathrm{~K}_{2} \mathrm{O}$ & 94,20 & 197,41 & 100,35 & 0,45 & 0,27 \\
$\mathrm{CaO}$ & 56,08 & 66,90 & 48,51 & 0,15 & 0,13 \\
$\mathrm{Na}_{2} \mathrm{O}$ & 61,98 & 19,31 & 11,43 & 0,04 & 0,03 \\
Total & - & 440,73 & 366,51 & 1,00 & 1,00 \\
\hline
\end{tabular}

Fonte: Elaboração própria.

A exergia química dos componentes inorgânicos do dejeto bovino, tanto do afluente quanto do efluente, foram estimados baseando-se na Tabela 2, utilizando a equação (2.1 e 6.1). Esses resultados estão apresentados nas tabelas 3 e 4. 
A Tabela 3 mostra que um $1 \mathrm{~kg}$ do dejeto analisado contém 16,702 MJ de exergia química até entrar em equilíbrio com o ambiente de referência, representando cerca de 23,72\% do poder calorífico de dejetos bovinos (13.500 kJ/kg), conforme FontPalma (2019). Desse valor, 99,24\% se deve a contribuição da matéria orgânica, calculada a partir da DQO. E dentre os componentes inorgânico, aquele que apresenta a maior contribuição foi o amônio $\left(\mathrm{NH}_{4}^{+}\right)$com $0,9 \%$, devido ao seu alto teor exergético, seguida do óxido de potássio $\left(\mathrm{K}_{2} \mathrm{O}\right)$ com $0,26 \%$.

Tabela 3 - Exergia química do dejeto bovino fresco em base seca.

\begin{tabular}{|c|c|c|c|c|c|}
\hline \multicolumn{6}{|c|}{ Exergia do Afluente } \\
\hline Parâmetros & $\begin{array}{c}\boldsymbol{b}^{\circ}{ }_{c h} \\
{[\mathbf{k J} / \mathbf{m o l}}\end{array}$ & $\begin{array}{l}\sum x_{i} b^{\circ}{ }_{c h_{i}} \\
{[\mathrm{~kJ} / \mathrm{mol}]}\end{array}$ & $\begin{array}{c}\sum R T_{0} x_{i} \ln x_{i} \\
{[\mathrm{~kJ} / \mathrm{mol}]}\end{array}$ & $\begin{array}{c}\boldsymbol{b}_{\text {ch }}^{\circ} \text { mistura } \\
{[\mathrm{kJ} / \mathrm{mol}]}\end{array}$ & $b^{\circ}{ }_{\text {ch }}{ }_{\text {total }}[\mathrm{kJ} / \mathrm{kg}]$ \\
\hline$D Q O$ & - & - & - & - & $16.575,86$ \\
\hline $\mathrm{NH}_{4}^{+}$ & 393,14 & 122,38 & $-0,90$ & 121,48 & \\
\hline $\mathrm{P}_{2} \mathrm{O}_{5}$ & 767,70 & 34,69 & $-0,35$ & 34,34 & \\
\hline $\mathrm{K}_{2} \mathrm{O}$ & 413,10 & 185,03 & $-0,89$ & 184,14 & 126,37 \\
\hline $\mathrm{CaO}$ & 127,30 & 19,32 & $-0,71$ & 18,61 & \\
\hline $\mathrm{Na}_{2} \mathrm{O}$ & 296,20 & 12,98 & $-0,34$ & 12,64 & \\
\hline Total & - & 374,40 & $-3,19$ & $8,27 *$ & $16.702,23$ \\
\hline
\end{tabular}

* A exergia química total da mistura está multiplicada pelo teor de sólidos fixos (SF), expresso na tabela1. Fonte: Elaboração própria.

A Tabela 4 evidencia que o componente orgânico da exergia química do efluente tratado (Digestato) possui uma contribuição de $98,40 \%$. O amônio $\left(\mathrm{NH}_{4}^{+}\right)$é o componente inorgânico que apresenta a maior contribuição com $2,55 \%$, seguida do óxido de potássio $\left(K_{2} O\right)$ com $0,27 \%$.

Além disso, observa-se que um $1 \mathrm{~kg}$ de efluente tratado ainda possui um conteúdo exergético de 15,35 MJ. Também podemos observar a destruição de 3,19 kJ/mol de exergia do afluente (tabela 3) e 3,00 kJ/mol do Digestato (Tabela 4), pelo processo de dissolução, descrita pelo segundo termo da equação (2.1) (Palacios-Bereche et al.,2013).

Tabela 4 - Exergia química do efluente tratado em base seca.

\begin{tabular}{|c|c|c|c|c|c|}
\hline \multicolumn{6}{|c|}{ Exergia do Efluente } \\
\hline Parâmetros & $\begin{array}{c}\boldsymbol{b}_{c h}^{\circ} \text { tabelada } \\
{[\mathrm{kJ} / \mathrm{mol}}\end{array}$ & $\begin{array}{l}\sum \boldsymbol{x}_{\boldsymbol{i}} \boldsymbol{b}^{\circ}{ }_{c h_{i}} \\
{[\mathrm{~kJ} / \mathrm{mol}]}\end{array}$ & $\begin{array}{c}\sum R T_{0} x_{i} \ln x_{i} \\
{[\mathrm{~kJ} / \mathrm{mol}]}\end{array}$ & $\begin{array}{c}\boldsymbol{b}^{\circ}{ }_{\text {ch }} \text { mistura } \\
{[\mathrm{kJ} / \mathrm{mol}]}\end{array}$ & $b^{\circ}{ }_{\text {ch }}{ }_{\text {total }}[\mathrm{kJ} / \mathbf{k g}]$ \\
\hline$D Q O$ & - & - & - & - & $15.105,01$ \\
\hline $\mathrm{NH}_{4}^{+}$ & 393,14 & 203,67 & $-0,84$ & 202,83 & \\
\hline $\mathrm{P}_{2} \mathrm{O}_{5}$ & 382,94 & 17,08 & $-0,34$ & 16,73 & \\
\hline $\mathrm{K}_{2} \mathrm{O}$ & 413,10 & 113,11 & $-0,88$ & 112,23 & 244,95 \\
\hline $\mathrm{CaO}$ & 127,30 & 16,85 & $-0,66$ & 16,18 & \\
\hline $\mathrm{Na}_{2} \mathrm{O}$ & 296,20 & 9,24 & $-0,27$ & 8,97 & \\
\hline Total & - & 359,94 & $-3,00$ & $12,45 *$ & $15.349,96$ \\
\hline
\end{tabular}

* A exergia química total da mistura está multiplicada pelo teor de sólidos fixos (SF), expresso na tabela1. Fonte: Elaboração própria. 
Com base na composição elementar média do estrume bovino presentes na literatura, estimou-se a exergia química dos componentes orgânicos pelos métodos de Peduzzi et al. (2016) e Shieh e Fan (1982), conforme ilustrado na Tabela 5. Desse modo, constatou-se que a exergia química da matéria orgânica, seguindo o método de Nakashima e Oliveira (2019), apresenta uma boa correspondência com a exergia calculada por outros métodos, estando dentro do intervalo de valores de 12.901,17 a $20.437,18 \mathrm{~kJ} / \mathrm{kg}$.

Tabela 5 - Análise elementar e exergia química da matéria orgânica presentes na literatura.

\begin{tabular}{|c|c|c|c|c|c|c|c|c|}
\hline & $\mathbf{C}$ & $\mathbf{H}$ & $\mathbf{O}$ & $\mathbf{N}$ & $\mathbf{S}$ & $\begin{array}{l}\text { Peduzzi et al. } \\
\text { (2016) }\end{array}$ & $\begin{array}{c}\text { Shieh \&Fan } \\
\text { (1982) }\end{array}$ & $\begin{array}{c}\text { Nakashima } \\
\text { (2019) }\end{array}$ \\
\hline & & & & & & \multicolumn{3}{|c|}{$\mathbf{b}^{\circ} \operatorname{ch}[\mathrm{kJ} / \mathrm{kg}]$} \\
\hline Itoh et al. (2019) & 50,80 & 6,20 & 40,00 & 2,50 & 0,50 & $13.460,81$ & $20.437,18$ & \\
\hline Chen et al. (1985) & 42,90 & 5,80 & 35,50 & 3,40 & 0,60 & $12.901,17$ & $17.611,39$ & $16.575,86$ \\
\hline Choi et al. (2014) & 38,06 & 5,18 & 28,15 & 1,85 & 0,25 & $14.317,32$ & $15.979,56$ & \\
\hline Wang et al. (2011) & 49,38 & 6,46 & 39,79 & 3,33 & 1,05 & $13.597,23$ & $20.273,22$ & \\
\hline "Máximo & 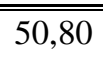 & 6,46 & 40,00 & 3,40 & 1,05 & 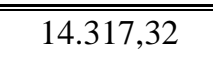 & $20.437,18$ & \\
\hline Médio & 45,29 & 5,91 & 35,86 & 2,77 & 0,60 & $13.569,13$ & $18.575,34$ & - \\
\hline Mínimo & 38,06 & 5,18 & 28,15 & 1,85 & 0,25 & $12.901,17$ & $15.979,56$ & \\
\hline Desvio Padrão & 5,13 & 0,48 & 4,80 & 0,64 & 0,29 & 504,60 & $1.871,93$ & \\
\hline
\end{tabular}

Fonte: Elaboração própria.

A exergia química específica da matéria inorgânica, calculadas pela equação (2.1), é 126,37 e 244,95 kJ/kg para o substrato e digestato, respectivamente. Estes resultados estão dentro do intervalo de valores presentes na literatura para resíduos sólidos urbanos, de 100 a $3.300 \mathrm{~kJ} / \mathrm{kg}$, conforme Song et al. (2013) e Eboh et al. (2016).

O aumento da exergia específica inorgânica do digestato se justifica devido a mineralizada da matéria orgânica, principalmente do nitrogênio orgânico. E quando comparado a exergia específica da matéria orgânica (16.702,23 e 15.349,96 $\mathrm{kJ} / \mathrm{kg}$ ), a exergia dos componentes inorgânicos é bem pequena, podendo ser desprezada. Contudo, esses valores ainda podem ser utilizados para calcular o custo evitado e o consumo específico de exergia dos biofertilizantes, auxiliando na identificação do potencial de economia de energia desses segmentos em comparação aos fertilizantes minerais.

Com base nos dados coletados em campo, com o auxílio do Landtec gem 5000 e confirmadas pela cromatografia gasosa, determinou-se as frações molares do biogás $\left(x_{j}\right)$, apresentados na Tabela 6 . A partir desses valores, estimou-se a exergia química do biogás em 29,55 MJ/m³ utilizando a equação (2.2), ilustrado na Tabela 7. Esse resultado corrobora com os dados publicados por Barati et al. (2017), com teor exergético de $26,73 \mathrm{MJ} / \mathrm{m}^{3}$, o que reflete em uma diferença percentual de 10,55\%. Observa-se também uma destruição de $1,31 \mathrm{~kJ} / \mathrm{mol}$ de exergia do biogás pelo processo de dissolução, até que este entre em equilíbrio com o ambiente de referência. 
Tabela 6 - Fração molar do Biogás.

\begin{tabular}{cccccc}
\hline $\begin{array}{c}\text { Espécie } \\
\text { Química }\end{array}$ & Unidades & $\begin{array}{c}\text { Massa Molar } \\
{[\mathbf{g} / \mathbf{m o l}]}\end{array}$ & $\begin{array}{c}\text { Valor } \\
\text { Experimental }\end{array}$ & $\begin{array}{c}\text { Fração Molar } \\
\left(\boldsymbol{x}_{\boldsymbol{j}}\right)\end{array}$ & $\begin{array}{c}\text { Densidade } \\
{\left[\mathbf{k g} / \mathbf{m}^{3}\right]}\end{array}$ \\
\hline $\mathrm{CH}_{4}$ & $\%$ & 16,04 & 58,5 & 0,79 & \\
$\mathrm{CO}_{2}$ & $\%$ & 44,01 & 41,3 & 0,20 & \\
$\mathrm{CO}$ & $\mathrm{ppm}$ & 28,01 & 14 & 0,000011 & \\
$\mathrm{O}_{2}$ & $\%$ & 15,999 & 0,2 & 0,0027 & - \\
$\mathrm{H}_{2} \mathrm{~S}$ & $\mathrm{ppm}$ & 98,079 & 1089 & 0,00024 & \\
$\mathrm{NH}_{3} *$ & $\%$ & 17,031 & 0,1 & 0,00000013 & \\
Total & - & - & - & 1,00 & 1,31 \\
\hline
\end{tabular}

* O valor da Amônia $\left(\mathrm{NH}_{3}\right)$ é um resultado médio coletado no trabalho de Deublein, Steinhauser (2011), p. 55-443. Fonte: Elaboração própria.

Tabela 7 - Exergia química do biogás.

\begin{tabular}{|c|c|c|c|c|c|c|}
\hline \multicolumn{7}{|c|}{ Exergia do Biogás } \\
\hline $\begin{array}{l}\text { Espécie } \\
\text { Química }\end{array}$ & $\begin{array}{c}\boldsymbol{b}^{\circ}{ }_{c h} \text { tabelada } \\
{[\mathrm{kJ} / \mathrm{mol}}\end{array}$ & $\begin{array}{l}\sum \boldsymbol{x}_{\boldsymbol{i}} \boldsymbol{b}^{\circ}{ }_{c h_{i}} \\
{[\mathrm{~kJ} / \mathrm{mol}]}\end{array}$ & $\begin{array}{c}\sum R T_{0} x_{i} \ln x_{i} \\
{[\mathrm{~kJ} / \mathrm{mol}]}\end{array}$ & $\begin{array}{l}b^{\circ}{ }_{\text {ch }} \text { total } \\
{[\mathrm{kJ} / \mathrm{mol}]}\end{array}$ & $\begin{array}{l}\boldsymbol{b}^{\circ}{ }_{\text {ch }} \text { total } \\
{\left[\mathbf{M J} / \mathbf{m}^{3}\right]}\end{array}$ & $\begin{array}{r}b^{\circ}{ }_{\text {ch }} \text { total } \\
{[\mathrm{kJ} / \mathrm{kg}]}\end{array}$ \\
\hline $\mathrm{CH}_{4}$ & 831,20 & 659,13 & $-0,46$ & 658,68 & & \\
\hline $\mathrm{CO}_{2}$ & 19,48 & 3,97 & $-0,80$ & 3,17 & & \\
\hline $\mathrm{CO}$ & 274,71 & 0,00 & $-0,00031$ & 0,0027 & & \\
\hline $\mathrm{O}_{2}$ & 3,97 & 0,01 & $-0,04$ & $-0,03$ & 29,55 & $22.516,04$ \\
\hline $\mathrm{H}_{2} \mathrm{~S}$ & 812,00 & 0,20 & 0,00 & 0,19 & & \\
\hline $\mathrm{NH}_{3}$ & 337,90 & 0,00 & $-0,000005$ & 0,00004 & & \\
\hline Total & - & 663,32 & $-1,31$ & 662,01 & & \\
\hline
\end{tabular}

Fonte: Elaboração própria.

\subsection{Análise do balanço exergético}

O acompanhamento dos dados levantados em campo e divulgados pelo CIBIOGÁS nos anos de 2016 a 2018, foram utilizados no balanço exergético do biodigestor, mostrado na Figura 1. Esta revela que o tratamento dos $200 \mathrm{~m}^{3}$ de resíduos da atividade leiteira, com produção média de $600 \mathrm{~m}^{3}$ de biogás por dia, possui um teor exergético de 64,97 GJ no dejeto fresco, podendo produzir 17,73 GJ de exergia na forma de biogás e 29,58 GJ como digestato. Assim, constata-se que a exergia contida no estrume é principalmente conservada no biofertilizante, o qual pode ser reutilizado nas culturas agrícolas, e parte dela é convertida em biogás. 
Figura 1 - Diagrama de Sankey para o balanço exergético do biodigestor anaeróbico.

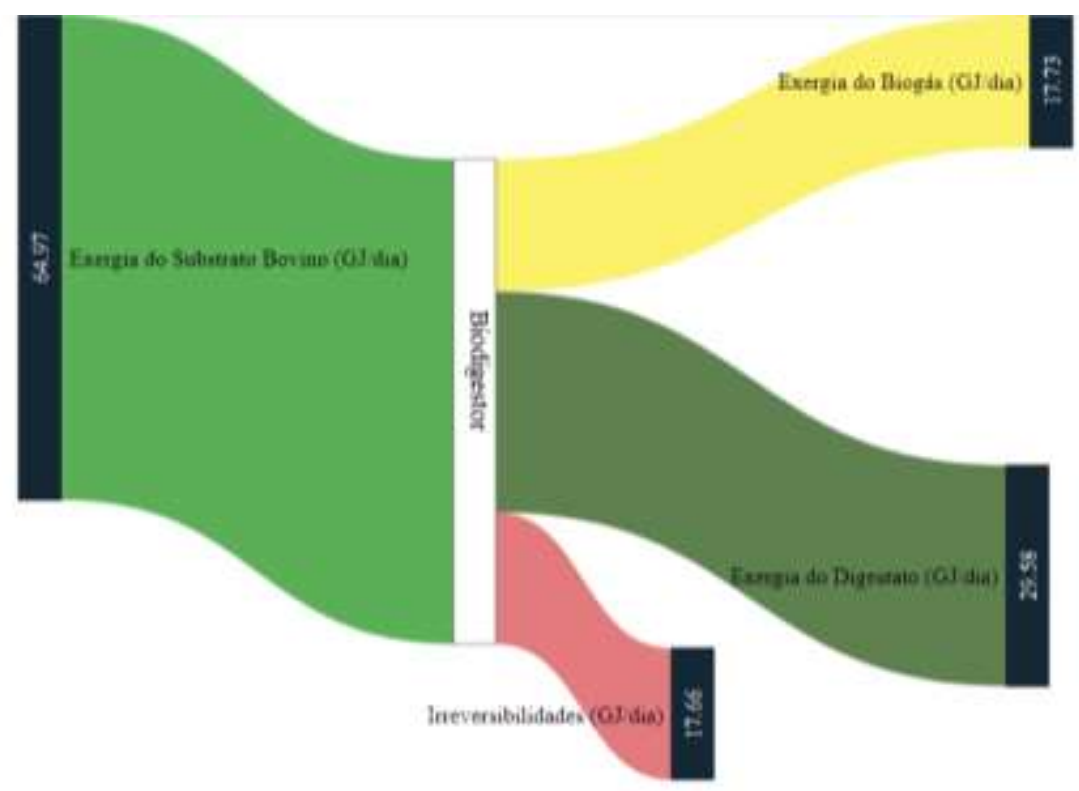

Fonte: Elaboração própria.

A irreversibilidade envolvida no processo de digestão (17,66 GJ/dia) foi atribuída às reações bioquímicas presentes na degradação anaeróbia da matéria orgânica e pela dissipação de calor devido à natureza exotérmica das reações. (Palacios-Bereche et al.,2013). Portanto, a avaliação da exergia física pode oferecer mais detalhes das irreversibilidades do processo, necessitando de um rigoroso acompanhamento da variação de temperatura da planta.

\subsection{Análise de eficiência exergética}

Com base nas equações (9.1), (9.2) e (9.3) foram analisadas as eficiências exergéticas, considerando as exergias dos produtos úteis por insumos consumidos. Esses resultados estão ilustrados na figura 2.

Como visto no gráfico da Figura 2, a eficiência exergética global do processo de digestão anaeróbia é bastante alta de $72,82 \%$. Constatando-se que o tratamento anaeróbio possui uma boa eficiência em reduzir a parcela de exergia química do dejeto fresco e produzir biogás durante o processo. Esse resultado é bem próximo a eficiência do tratamento anaeróbio divulgadas pelo CIBIOGÁS no ano de 2017, que no caso foi de 75\%, considerando a redução de carga orgânica e produção de biogás (Biasi et al.,2018). 
Figura 2 - Eficiência Exergética para os diferentes níveis de agregação.

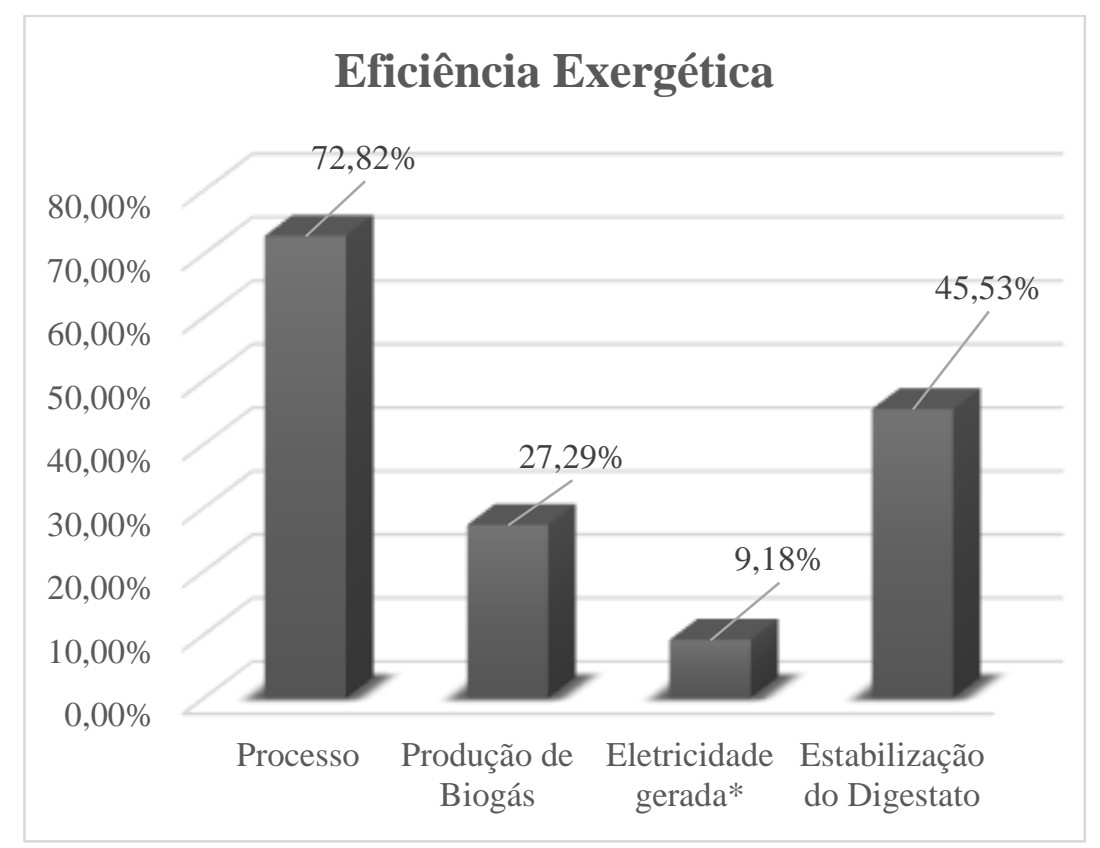

* Este resultado considera a eficiência do grupo gerador da Starmilk, levantadas por Brandt (2019). Fonte: Elaboração própria.

No entanto, esse valor de eficiência, superestima o desempenho do processo, no que se refere a produção de biogás. Desse modo, quando o biogás é considerado como o único produto útil do sistema, em termos de produção de energia, a eficiência fica em torno de 27,29\%. Resultado inferior ao encontrado nos trabalhos de Barreira et al. (2016), Barati et al. (2017) e Xydis et al. (2013), o qual registram eficiência exergética de produção de biogás bruto de 54,11\%,33,14\% e 33\% em reatores alimentados com vinhaça e resíduos sólidos urbanos, respectivamente. Essa baixa eficiência de produção de biogás é principalmente atribuída a operação e alimentação dos biodigestores, que de acordo com Vandevivere et al. (2002), resíduos com alto teor de fibra lignocelulósica, como a maravalha utilizada na cama animal da Starmilk, possui um baixo rendimento de biogás caso não utilize biodigestores robustos, com sistemas de aquecimento, agitação e multiestágios.

Considerando esse valor de eficiência de produção de biogás (27,29\%), a exergia específica total, calculada pela equação (6) $\left(324,84 \mathrm{MJ} / \mathrm{m}^{3}\right)$, também mostra que o dejeto analisado tem potencial de produzir $90,01 \mathrm{MJ}$ de energia por $\mathrm{m}^{3}$ de dejeto bovino, na forma de biogás. Caso considere a eficiência do grupo gerador da Starmilk em 33,62\% (Brandt, 2019), o dejeto analisado tem potencial de gerar $8,28 \mathrm{kWh}$ de eletricidade por $\mathrm{m}^{3}$ de dejeto bovino. Em 2017, o CIBiogás relatou um rendimento produção de 4,20 kwh/m³ de dejeto na Starmilk (Biasi et al.,2018), o que reflete em 97,15\% da estimativa exergética realizada neste estudo. Contudo, é importante salientar que a estimativa exergética não pode ser atingida pelo fato de considerar apenas processos reversíveis, servindo apenas como limite teórico de desempenho termodinâmico possível. Em outros estudos, Barrera et al. (2016) e Nakashima e Oliveira (2019), relataram rendimento de produção de 26,6 kWh/m³ e $21 \mathrm{kWh} / \mathrm{m}^{3}$, respectivamente, para usinas de biogás tratada com vinhaça, reforçando que a planta da Starmilk ainda pode sofre melhorias.

Quando consideramos a eficiência em estabilizar os componentes químicos do dejeto bovino, em que a exergia do efluente tratado é tida como o único produto útil, a eficiência encontrada foi de $43,95 \%$. Valor esse que contabiliza não só a redução de carga orgânica de 51,85\%, determinada pela DQO, mas também a mineralização do material inorgânico.

\section{Conclusão}

Neste estudo foi aplicado uma metodologia para estimar a exergia química $\left(b^{\circ}{ }_{c h}\right)$, bem como, a eficiência do processo de tratamento de dejetos, partindo de parâmetros físico-químicos comumente utilizados na avaliação de águas residuárias. 
Nesse caso, o tratamento dos dejetos bovinos, com teor de exergia bruta de 64,97 GJ/dia, tem potencial de gerar 17,73 $\mathrm{GJ} /$ dia de exergia na forma de biogás, 29,58 GJ/dia como digestato e perdendo como irreversibilidade 17,66 GJ/dia durante o processo. Assim, infere-se uma eficiência exergética de 45,53\% na estabilização dos compostos químicos, podendo recuperar até $27,29 \%$ da exergia do estrume bovino na forma de biogás.

A metodologia desenvolvida é particularmente útil para analisar projetos que integre biodigestores com demais sistemas, como moto gerador, fertirrigação ou mesmo purificação do biogás. Por esse motivo, a análise termodinâmica do processo de digestão considerou os componentes inorgânicos, úteis na fertilização de culturas agrícolas, bem como, todos os gases presentes no biogás, o que possibilita uma avaliação da eficiência dos filtros purificadores. Portanto, a análise exergética apresentada aqui pode fornecer diretrizes e planos metodológicos importantes para investigações futuras, a fim de desenvolver plantas de tratamento de resíduos e produção de biogás termodinamicamente e ambientalmente eficientes.

\section{Agradecimentos}

O presente trabalho foi realizado com apoio da Coordenação de Aperfeiçoamento de Pessoal de Nível Superior Brasil (CAPES) - Código de Financiamento 001. Agradecimentos especiais ao professor Dr. Jair Antônio Cruz Siqueira pelo incentivo a pesquisa, ao apoio nas amostragens da mestranda Cristela Marta Siebert e aos esclarecimentos e sugestões do professor Dr. Armin Feiden.

\section{Referências}

Barati, M. R., Aghbashlo, M., Ghanavati, H., Tabatabaei, M., Sharifi, M., Javadirad, G., Dadak, A., \& Mojarab Soufiyan, M. (2017). Comprehensive exergy analysis of a gas engine-equipped anaerobic digestion plant producing electricity and biofertilizer from organic fraction of municipal solid waste. Energy Conversion and Management, 151, 753-763. https://doi.org/10.1016/j.enconman.2017.09.017.

Barrera, E. L., Rosa, E., Spanjers, H., Romero, O., de Meester, S., \& Dewulf, J. (2016). A comparative assessment of anaerobic digestion power plants as alternative to lagoons for vinasse treatment: life cycle assessment and exergy analysis. Journal of Cleaner Production, 113, 459-471. https://doi.org/10.1016/j.jclepro.2015.11.095.

Campos, A. T. de, Campos, A. T. Campos, D. S., \& Pires, M. de F. A. (2003). Tratamento e reciclagem de águas residuárias em sistema intensivo de produção de leite. Circular Técnica n. 75. Juiz de Fora: Embrapa Gado de Leite. http://ainfo.cnptia.embrapa.br/digital/bitstream/item/65245/1/CT-75-Tratamento-ereciclagem-de-aguas.pdf.

Chen, L., Mehta, C., Ishimi, T., Fan, L., \& Chen, Y. (1985). Thermodynamic analysis of anaerobic digestion of cattle manure. Agricultural Wastes, 14(2), 7996. https://doi.org/10.1016/s0141-4607(85)80021-4.

Choi, H. L., Sudiarto, S. I., \& Renggaman, A. (2014). Prediction of livestock manure and mixture higher heating value based on fundamental analysis. Fuel, 116, 772-780. https://doi.org/10.1016/j.fuel.2013.08.064.

CIBIOGÁS, Centro Internacional de Energias Renováveis. (2016). Análise físico-química dos efluentes da Fazenda Iguaçu StarMilk. Fundação Parque Tecnológico de Itaipu.

CIBIOGÁS, Centro Internacional de Energias Renováveis. (2017). Análise físico-química dos efluentes da Fazenda Iguaçu StarMilk. Fundação Parque Tecnológico de Itaipu.

CIBIOGÁS, Centro Internacional de Energias Renováveis. (2018). Análise físico-química dos efluentes da Fazenda Iguaçu StarMilk. Fundação Parque Tecnológico de Itaipu.

CONAB-Companhia Nacional de Abastecimento. (2020). Análise mensal brasileiras. Leite e Derivados, Brasília.

Deublein D., \& Steinhauser A. (2011). Biogas from Waste and Renewable Resources: an Introduction. John Wiley \& Sons.

Estrela, C. (2018). Metodologia Científica: Ciência, Ensino, Pesquisa. Editora Artes Médicas.

Font-Palma, C. (2019). Methods for the Treatment of Cattle Manure-A Review. C, 5(2), 27. https://doi.org/10.3390/c5020027.

Itoh, T., Iwabuchi, K., Maemoku, N., Sasaki, I., \& Taniguro, K. (2019). A new torrefaction system employing spontaneous self-heating of livestock manure under elevated pressure. Waste Management, 85, 66-72. https://doi.org/10.1016/j.wasman.2018.12.018.

Khosravi, S., Panjeshahi, M. H., \& Ataei, A. (2013). Application of exergy analysis for quantification and optimisation of the environmental performance in wastewater treatment plants. International Journal of Exergy, 12(1), 119. https://doi.org/10.1504/ijex.2013.052552. 
Kobayashi, N., Noel, E. A., Barnes, A., Watson, A., Rosenberg, J. N., Erickson, G., \& Oyler, G. A. (2013). Characterization of three Chlorella sorokiniana strains in anaerobic digested effluent from cattle manure. Bioresour. Technol. 150(3). 77-386.

Kotas T. J. (2013). The Exergy Method of Thermal Plant Analysis, Elsevier.

Kunz, A., \& Oliveira, P. A. V. (2006). Aproveitamento de dejetos de animais para geração de biogás. Revista de Política Agrícola 15 (3) $28-35$.

Kunz, A, Steinmetz, R. L. R, Amaral, A. C. (2019). Fundamentos da digestão anaeróbia, purificação do biogás, uso e tratamento do digestato. Embrapa Suínos e Aves. Concordia SC. (pp.209). http://ainfo.cnptia.embrapa.br/digital/bitstream/item/197183/1/Livro-Biogas.pdf.

Lorimor, J. C., Powers, W. J., \& Sutton, A. 1. (2004). Manure Characteristics. MidWest Plan Service, Iowa State University, 24.

Lozano, M., \& Valero, A. (1993). Theory of the exergetic cost. Energy, 18(9), 939-960. https://doi.org/10.1016/0360-5442(93)90006-y.

Manitoba. (2015). Properties of Manure, Final Report for the Manitoba Livestock Manure. Management Initiative.

Nakashima, R., \& de Oliveira Junior, S. (2020). Comparative exergy assessment of vinasse disposal alternatives: Concentration, anaerobic digestion and fertirrigation. Renewable Energy, 147, 1969-1978. https://doi.org/10.1016/j.renene.2019.09.124.

Nitsche, P. R., Caramori, P. H., Ricce, W. S., \& Pinto, L. F. D. (2019). Atlas Climático do Estado do Paraná, Londrina, PR: IAPAR.

Palacios-Bereche, R., Mosqueira-Salazar, K. J., Modesto, M., Ensinas, A. V., Nebra, S. A., Serra, L. M., \& Lozano, M. A. (2013). Exergetic analysis of the integrated first- and second-generation ethanol production from sugarcane. Energy, 62, 46-61. https://doi.org/10.1016/j.energy.2013.05.010.

Peduzzi, E., Boissonnet, G., \& Maréchal, F. (2016). Biomass modelling: Estimating thermodynamic properties from the elemental composition. Fuel, 181, 207217. https://doi.org/10.1016/j.fuel.2016.04.111.

Peixoto, G., Saavedra, N. K., Varesche, M. B. A., \& Zaiat, M. (2011). Hydrogen production from soft-drink wastewater in an upflow anaerobic packed-bed reactor. International Journal of Hydrogen Energy, 36(15), 8953-8966. https://doi.org/10.1016/j.ijhydene.2011.05.014.

Souza, R. C. P., et al. (2018). Análise exergética da vinhaça para produção de biogás, 2018. Departamento Acadêmico de Engenharia Ambiental. Universidade Tecnológica Federal do Paraná, Londrina.

Szargut J., \& Egzergia. (2007) Exergy method: technical and ecological application. Publishing House of the Silesian University of Technology. Gliwice, Polond. Szargut, J, Morris, D. R, \& Steward, F. R. (1988). Exergy analysis of thermal, chemical, and metallurgical processes. Hemisphere Publishing, New York, NY.

Tai, S., Matsushige, K., \& Goda, T. (1986). Chemical exergy of organic matter in wastewater. International Journal of Environmental Studies, 27(3-4), 301315. https://doi.org/10.1080/00207238608710299.

Tambone, F., Genevini, P., D’Imporzano, G., \& Adani, F. (2009). Assessing amendment properties of digestate by studying the organic matter composition and the degree of biological stability during the anaerobic digestion of the organic fraction of MSW. Bioresource Technology, 100(12), 3140-3142. https://doi.org/10.1016/j.biortech.2009.02.012.

Vandevivere, P., De Baere, L., \& Verstraete, W. (2002). Types of Anaerobic Digester for Solid Wastes. In: Mata-Alvarez, J. (2002). Biomethanization of the Organic Fraction of Municipal Solid. Wastes. Water Intelligence Online, Publishing J. Londres, 4(0) 111-137.

Wang, L., Shahbazi, A., \& Hanna, M. A. (2011). Characterization of corn stover, distiller grains and cattle manure for thermochemical conversion. Biomass and Bioenergy, 35(1), 171-178. https://doi.org/10.1016/j.biombioe.2010.08.018.

Xydis, G., Nanaki, E., \& Koroneos, C. (2013). Exergy analysis of biogas production from a municipal solid waste landfill. Sustainable Energy Technologies and Assessments, 4, 20-28. https://doi.org/10.1016/j.seta.2013.08.003. 